\title{
Über das Treiben von Wurzeln
}

\author{
Von \\ Hans Molisch \\ w. M. k. Akad.
}

Aus dem Pflanzenphysiologischen Institut der k. k. Universität in Wien. Nr. 100 der zweiten Folge

(Mit 2 Tafeln)

(Vorgelegt in der Sitzung am 18. Jänner 1917)

I.

Die Ruheperiode der Pflanze steht gegenwärtig im Vordergrunde des Interesses. In den letzten zwei bis drei Dezennien sind mehrere ausgezeichnete Mittel bekannt geworden, durch die die Ruheperiode der Knospen aufgehoben werden kann, so daß sie zu ganz ungewohnten Zeiten zum Austreiben gebracht werden können.

$\mathrm{Da}$ ich die Literatur dieses Gegenstandes schon zu wiederholten Malen berührt habe ${ }^{1}$ und da erst vor kurzem zusammenfassende Berichte von Kniep ${ }^{2}$ und Lakon ${ }^{3}$ darüber erschienen sind, so sei darauf verwiesen.

Auffallenderweise hat man dabei ausschließlich die Ruheperiode und das Treiben der Blatt- und Blütenknospen in Betracht gezogen, das Treiben der Wurzeln aber wurde bisher meines Wissens noch nie untersucht, ja die Frage, ob sich die Wurzeln auch treiben lassen, wurde überhaupt noch nie gestellt.

1 Molisch H., Pflanzenphysiologie als Theorie der Gärtnerei. Jena 1916, p. $160-174$.

2 Kniep H., Über rhythmische Lebensvorgänge bei den Pflanzen. Sammelref., Verhandl. d. phys.-med. Ges. zu Würzburg, 1915, 23 S.

3 Lakon S., Über den rhythmischen Wechsel von Wachstum und Ruhe beiden Pflanzen. Biol. Ztbl., 1915, p. $401-471$. 
Die Periodizität der Wurzelbildung bei ausdauernden Gewächsen ist noch wenig erforscht; es ist dies auch nur zu begreiflich, da die Beobachtung der gewöhnlich in der Erde befindlichen Wurzeln Schwierigkeiten darbietet. Die Resultate, zu denen verschiedene Forscher (Resa, Wieler, Rimbach, Büsgen, Hämmerle und A. Engler ${ }^{1}$ ) bezüglich der Periodizität der Wurzeln gelangten, sind sogar in den Hauptpunkten keineswegs übereinstimmend. Nur soviel scheint mit einiger Sicherheit hervorzugehen, daß viele Wurzeln schon im März mit ihrem Wachstum beginnen und es dann bis in den November oder Dezember fortsetzen. Im Nachsommer dürfte eine Verzögerung des Wachstums, aber kein völliger Stillstand platzgreifen. Aus den Beobachtungen, die bis heute vorliegen, geht aber nicht hervor, ob den Wurzeln überhaupt eine freiwillige-Ruhe zukommt oder ob sie im Winter nur deshalb nicht wachsen, weil sie ungünstigen Wachstumsbedingungen, vor allem, weil sie in unseren Breiten niederer Temperatur ausgesetzt sind. Würde sich $z$. B. herausstellen, daß Zweige, die leicht Adventivwurzeln bilden, im Herbst oder Winter diese Neigung trotz günstiger Wachstumsfaktoren nicht bekunden, wohl aber, wenn sie dem Warmbad ${ }^{2}$ oder dem Rauch ausgesetzt worden waren, so würde dies entschieden für eine freiwillige Ruhe sprechen. Solche Versuche habe ich schon vor zehn Jahren mit dem Warmbad gemacht, aber erst im Herbst und Winter 1916/17 wurden sie in größerer Zahl und mit verschiedenen Pflanzen systematisch durchgeführt.

Bezüglich der Methodik sei folgendes hervorgehoben: $\mathrm{Zu}$ den Versuchen dienten ausschließlich frisch abgeschnittene ein- bis dreijährige $Z$ weige von solchen Gehölzen, die im feuchten Raume leicht Adventivwurzeln bilden. Dazu gehören nach meinen und anderen Erfahrungen Salix-, Populus-Arten, Philadelphus coronarius, Viburnum opulus und, wenn auch weniger gut, Forsythia suspensa. Überrascht war ich von der Wurzelbildung von Viburnum opulus, weil hier die Wurzeln

1 Jost L., Vorlesungen über Pflanzenphysiologie, 2. Aufl., Jena 1908, p. 421 .

2 Molisch H., Das Warmbad als Mittel zum Treiben der Pflanzen. Jena 1909, p. 4. 
knapp unter den Knospen, und zwar auch unter den Terminalknospen hervorkommen (Fig. 4).

Die Zahl der Stoffe, die ruhende Knospen zum raschen Austreiben veranlassen können und die ich kurz als »Treibstoffe ${ }^{1}{ }^{1}$ bezeichnet habe, ist bereits eine verhältnismäßig große. In meinen Versuchen wurde entweder warmes Wasser oder Rauch von Tabak oder Papier angewendet. Die Warmbad- ${ }^{2}$ und Rauchmethode ${ }^{3}$ war ganz dieselbe, wie ich sie in meinen einschlägigen Abhandlungen beschrieben habe. Nach der Behandlung mit Rauch wurden die Zweige 1 bis 2 Stunden an die frische Luft ins Freie gebracht und dann zumeist im feuchten Warmhause im Licht bei einer Temperatur von 12 bis $20^{\circ}$, manchmal auch im Thermostaten bei etwa $25^{\circ} \mathrm{C}$. unter Glasstürzen im Finstern aufgestellt. Die Resultate waren im Gewächshause gewöhnlich auffallender, da hohe Temperatur und Lichtabschluß im Thermostaten den Einfluß des Bades oder Rauches, weil im gleichen Sinne wirkend, einigermaßen verschleiern.

\section{Versuche.}

\section{Versuch.}

\section{Philadelphus coronarius.}

Am 23. August 1916 wurden beblätterte $Z$ weige 24 Stunden im Rauch belassen. Es wurden zwei Bündel I und II zu je drei Zweigen verwendet.

Bündel I wurde nicht dem Rauche ausgesetzt (Kontrollversuch).

Bündel II wurde 24 Stunden im Tabakrauche belassen.

Nachher wurden die Zweige, in Wassergläsern unbedeckt stehend, im Warmhause am Lichte weiter kultiviert. Am 1. Oktober war der Unterschied auffallend. Die Rauchsprosse, die, nebenbei bemerkt, ihre Blätter viel früher verloren hatten

1 Molisch H., Über das Treiben ruhender Pflanzen mit Rauch. Diese Sitzungsber., Abt. I, 125. Bd., p. 157.

2 Molisch H., 1. c., p. 143.

3 Molisch H., 1. c., p. $13-18$. 
als die Kontrollsprosse, hatten oberhalb des Wassers einen ganzen Pelz von Wurzeln gebildet, während die Kontrollsprosse keine oder nur wenige ganz kurze Wurzeln erzeugt hatten (Fig. 1).

Aus diesem Versuche geht hervor, daß der Rauch schon zu einer Zeit, wenn die Blätter noch fest am Sprosse sitzen, das Austreiben der Wurzeln in hohem Grade beschleunigt.

\section{Versuch.}

\section{Philadelphus coronarius.}

13. X. 1916. Drei Bündel (I bis III) zu je drei Zweigen wurden verwendet.

Bündel I diente als Kontrolle.

Bündel II wurde 48 Stunden Papierrauch ausgesetzt.

Bündel III wurde 48 Stunden Tabakrauch ausgesetzt.

Nachher wurden alle Zweige im Warmhause am Lichte weiter kultiviert.

6. XI. 1916. Inzwischen waren alle Blätter abgefallen. Bei II und III früher als bei I.

Bei II und III kommen oberhalb des Wasserspiegels zahlreiche Wurzeln hervor, bei I nur wenige.

17. XI. 1916. Bei allen Zweigen haben sich zahlreiche Wurzeln entwickelt, bei II und III sind sie länger. Der Unterschied in der Wurzelentwicklung zwischen den Kontroll- und Rauchzweigen war zwar noch immer zu sehen, aber er war doch nicht so deutlich wie bei Versuch 1, wahrscheinlich weil die Ruheperiode schon im Ausklingen begriffen war, denn die Knospen der Kontrollzweige hatten auch schon ausgetrieben.

\section{Versuch.}

\section{Salix sp.}

Am 1. November 1908 wurden Zweige der Weide im Wasser von $30^{\circ}$ durch 12 Stunden gebadet und dann im Warmhause, diffus beleuchtet, aufgestellt. Nach 14 Tagen hatten die gebadeten Zweige, im Wasser stehend, zahlreiche 
lange Wurzeln gebildet, während die ungebadeten Kontrollzweige noch keine Wurzeln getrieben oder die Wurzelspitzen eben erst nach dem Durchbruch der Rinde hatten erscheinen lassen (Fig. 2).

\section{Versuch.}

Salix sp. und Philadelphus coronarius.

11. XI. 1916. Ein- bis Zweijährige Zweige von $35 \mathrm{~cm}$ Länge wurden 12 Stunden in Wasser von $30^{\circ}$ C. gebadet. Während des Bades waren sie vollständig untergetaucht. Die Kontrollzweige genossen während dieser Zeit dieselbe Temperatur, blieben aber in feuchter Luft. Nachher wurden beide Bündel (I und II) in großen Glaszylindern so untergebracht, daß nur das basale Ende im Wasser war, der übrige Teil des Zweiges befand sich in feuchter Luft. Die Glaszylinder waren mit Glasplatten zugedeckt. Die Temperatur war 15 bis $18^{\circ} \mathrm{C}$., die Beleuchtung mäßig.

23. XI. 1916.

Bündel I, nicht gebadet. Kein Treiben.

Bündel II, gebadet. Es haben sich zahlreiche, $1 / 2$ bis $3 \mathrm{~cm}$ lange Adventivwurzeln gebildet, auch die Laub- und Blütenknospen treiben.

7. XII. 1916.

Bündel I, nicht gebadet. Weder Knospen noch Wurzeln treiben.

Bündel II. Die Zweige haben 5 bis $10 \mathrm{~cm}$ lange Laubtriebe und reichlich 3 bis $7 \mathrm{~cm}$ lange Wurzeln gebildet.

Aus diesem Versuch geht deutlich hervor, daß das Warmbad nicht bloß die Knospen, sondern auch das Treiben der Wurzeln in hohem Grade beeinflußt.

Derselbe Versuch wurde auch gleichzeitig mit Philadelphus coronarius gemacht und gab gleichfalls ein positives Resultat. Die gebadeten Zweige trieben nach vier Wochen Knospen und Wurzeln, die ungebadeten aber nicht. 
Wurden die Versuche mit der Weide in der zweiten Hälfte Dezember oder später gemacht, so zeigt sich kein deutlicher Unterschied mehr zwischen gebadeten oder geräucherten $Z$ weigen, da die Ruheperiode für die Knospen und Wurzeln schon vorüber ist.

\section{Versuch.}

Populus nigra.

Am 26. Oktober 1916 wurden drei Bündel (I bis III) von je zwei Zweigen dem Versuche unterworfen.

Bündel I diente zur Kontrolle.

Bündel II wurde durch 24 Stunden dem Papierrauch ausgesetzt.

Bündel III wurde durch 24 Stunden dem Tabakrauch ausgesetzt.

Alle Zweige standen während der 24 stündigen Rauchwirkung im finsteren Thermostaten $\left(28^{\circ}\right)$, wurden dann im Freien durch 1 Stunde gelüftet und kamen dann wieder in den Thermostaten. Hier standen sie unter Glasstürzen, die mit Wasser abgesperrt waren.

31. X. Alle Blätter waren abgefallen, bei II und III auch einzelne Knospen. Auf den am Sprosse zurückbleibenden Blattund Knospennarben bilden die Zellen der Trennungsschichte einen mehlartigen Belag.

7. XI.

I unverändert.

II zahlreiche Wurzeln bereits 2 bis $5 \mathrm{~mm}$ lang.

III zahlreiche Wurzeln bereits 2 bis $3 \mathrm{~cm}$ lang.

10. XI.

I unverändert.

II zahlreiche Wurzeln schon $1 \mathrm{~cm}$ lang.

III zahlreiche Wurzeln schon 3 bis $5 \mathrm{~cm}$ lang.

Bei II und III trieben auch schon die Knospen. 
Je zwei Zweige von I und III wurden jetzt photographiert (Fig. 3). Nach 3 Tagen kamen auch bei I Wurzeln zum Vorschein.

18. XI. Die Rauchzweige (II und III) hatten inzwischen auch ihre Blatt- und Blütenknospen ausgetrieben, während die Kontrollzweige noch kein Treiben zeigten. Auch bezüglich des Wurzeltreibens war der Unterschied zwischen Kontroll- und Rauchzweigen in die Augen springend.

Bei Wiederholung der Versuche zeigte sich mit wenigen Ausnahmen dasselbe, auch war die Lentizellenwucherung der geräucherten oder gebadeten Zweige stärker als die der Kontrollexemplare.

\section{Versuch.}

\section{Viburnum opulus.}

Die Sprosse dieser Pflanze eignen sich in vorzüglicher Weise für unsere Versuche, da sie die interessante Eigentümlichkeit haben, im feuchten Raume knapp unter den Blattknospen reichlich Wurzeln $\mathrm{zu}$ bilden. ${ }^{1}$

2. XI. 1916. Drei Bündel I bis III zu je drei Zweigen.

Bündel I diente als Kontrolle.

Bündel II wurde 24 Stunden Papierrauch ausgesetzt.

Bündel III wurde 6 Stunden Tabakrauch ausgesetzt.

Nachher wurden die Zweige im Warmhause im Lichte weiter kultiviert.

17. XI. 1916.

Bündel I keine Wurzeln.

Bündel II mehrere Wurzeln, $1 / 4$ bis $1 / 2 \mathrm{~cm}$ lang.

Bündel III mehrere Wurzeln, $1 / 4$ bis $1 \mathrm{~cm}$ lang.

18. XII. 1916.

Bündel I keine Wurzeln, Knospen treiben nicht.

Bündel II viele 2 bis $4 \mathrm{~cm}$ lange Wurzeln, Knospen treiben.

1 Viburnum lantana zeigt diese Eigentümlichkeit nicht. 
Bündel III mehrere 1 bis $2 \mathrm{~cm}$ lange Wurzeln, Knospen treiben.

Die Fig. 4 zeigt den Unterschied zwischen I und II.

Also auch bei Viburnum opulus wird das Austreiben der Wurzeln durch Reizmittel beschleunigt.

Der vorhin geschilderte Versuch wurde zu der angegebenen Zeit mehrmals wiederholt und gab im wesentlichen dieselben Ergebnisse. Die Wurzeln trieben manchmal früher aus als die zugehörigen Knospen, häufig schlugen beide gleichzeitig oder die Wurzeln später aus.

Aus den Untersuchungen von Vöchting geht bereits hervor, daß in der Rinde der Weidenzweige Wurzelanlagen verborgen sind, die als schwach vorgewölbte Hügel unter der primären Rinde sitzen. "Mit ziemlicher Regelmäßigkeit findet man diese Adventiv-Wurzel-Anlagen auf beiden Seiten der Achselsprosse, gewöhnlich etwas tiefer als die untere Grenze der Ansatzstelle der letzteren. «1

Bei Viburmum opulus habe ich nach solchen Anlagen vergebens gesucht, doch ist es nicht unwahrscheinlich, daß der morphologische Ort der Adventiv-Wurzel-Anlage auch hier schon bestimmt, aber nur durch einige wenige Zellen repräsentiert ist, so daß die Anlage als solche nicht auffindbar ist.

Es ist bemerkenswert, daß bei den Viburmum-Sprossen die Wurzeln gewöhnlich knapp unter der Endknospe oder den höchsten Knospen zuerst und mit Vorliebe entstehen, gegen die Basis aber seltener.

Bekanntlich ist dies bei Weiden, Pappeln und anderen Gehölzen umgekehrt, denn hier brechen die Wurzeln, der Polarität entsprechend, am reichlichsten an den der Basis genäherten Stellen hervor.

Nach der gegebenen Sachlage müssen wir annehmen, daß die Treibstoffe auf die Wurzelanlagen, mögen sie deutlich oder undeutlich ausgebildet sein, in ähnlicher Weise wirken wie auf ruhende Knospen.

1 Vöchting H., Über Organbildung im Pflanzenreich. I. T., Bonn 1878 , p. 24. 
Auch die Wurzelanlagen der genannten Gewächse haben also eine Zeit, in der sie zum Auswachsen nicht oder weniger geneigt sind. Das ist die Zeit ihrer Ruhe, aus der sie sich durch Treibmittel wecken und zum Wachstum bewegen lassen.

\section{Zusammenfassung.}

Werden Zweige von Salix, Populus, Philadelphus coronarius und Viburmum opulus in den Monaten September, Oktober und November einem Warmbad oder dem Rauche von Papier oder Tabak in der beim Treiben von Laub- und Blütenknospen üblichen Weise ausgesetzt, so entstehen nachher an den gebadeten oder geräucherten Zweigen die Adventivwurzeln gewöhnlich bedeutend früher als an den unbehandelten Kontrollexemplaren. Es lassen sich also nicht bloß Laub- und Blütenknospen, sondern auch die Anlagen von Adventivwurzeln treiben.

Diese Tatsache spricht dafür, daß die mehrfach beobachtete Periodizität des Wurzelwachstums bei Gehölzen nicht immer eine unfreiwillige, durch ungünstige Wachstumsfaktoren hervorgerufene, sondern in vielen Fällen eine freiwillige sein dürfte wie die der herbstlichen Knospen unserer heimischen Bäume und Sträucher. 


\section{Erklärung der Photographien.}

\section{Tafel I.}

Fig. 1. Philadelphus coronarius. Rechts Zweige, die 24 Stunden Tabakrauch ausgesetzt waren, links die Kontrollzweige. Beginn des Versuches am 23. August, Ende am 1. Oktober. Die Rauchzweige treiben viel früher und reichlicher Wurzeln als die Kontroll$\mathrm{z}$ weige.

Fig. 2. Salix sp. Links Zweige, die durch 12 Stunden in Wasser von $30^{\circ}$ gebadet wurden, rechts die Kontrollzweige. Beginn des Versuches am 1. November 1908, Ende 15. November 1908. Die gebadeten Zweige treiben Laub-, Blütenknospen und zahlreiche Wurzeln, die ungebadeten beginnen am Ende des Versuches die Knospen erst zu strecken.

\section{Tafel II.}

Fig. 3. Populus nigra. Links Zweige, die durch 24 Stunden Tabakrauch ausgesetzt waren, rechts die Kontrollzweige. Beginn des Versuches am 26. Oktober, Ende am 10. November. Die Rauchzweige entwickelten reichlich Wurzeln, die Kontrollzweige nicht.

Fig. 4. Viburnum opulus. Links Zweige, die durch 24 Stunden Papierrauch ausgesetzt waren, rechts die Kontrollzweige. Beginn des Versuches am 2. November, Ende am 18. Dezember. Bei den Rauchzweigen treiben die Terminalknospen und vornehmlich in ihrer Nähe auch reichlich Wurzeln, bei den Kontrollzweigen hingegen noch kein Treiben. 


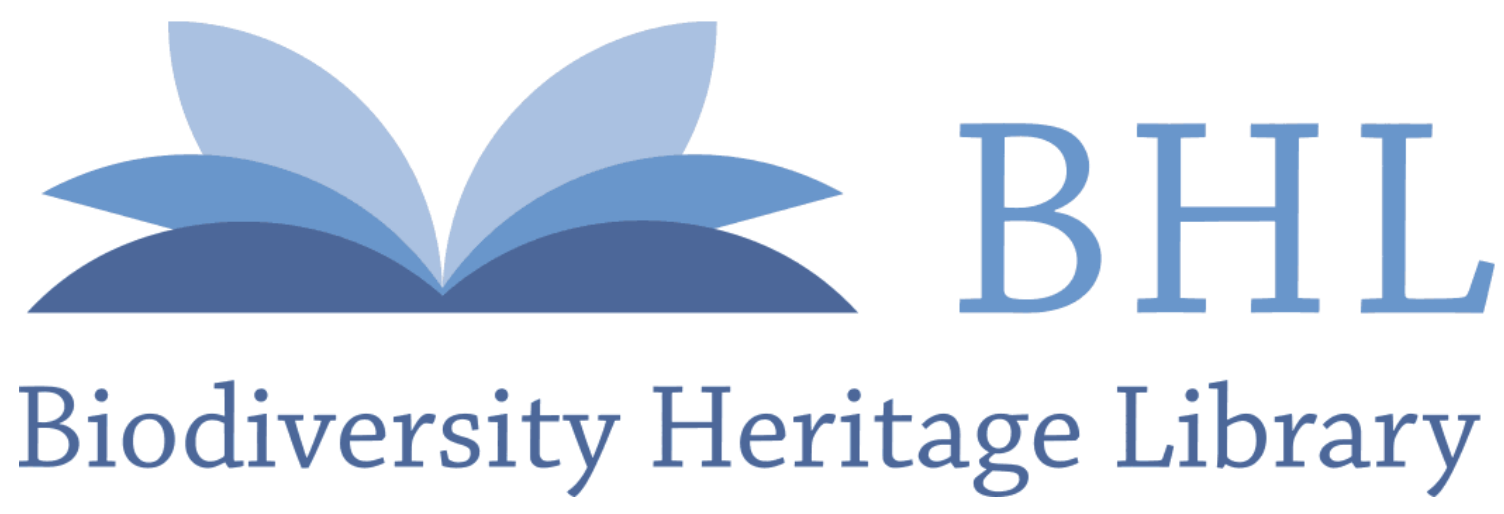

Molisch, Hans. 1917. "Über das Treiben von Wurzeln." Sitzungsberichte der Kaiserlichen Akademie der Wissenschaften. Mathematisch-Naturwissenschaftliche Klasse 126, 3-12.

View This Item Online: https://www.biodiversitylibrary.org/item/35552

Permalink: https://www.biodiversitylibrary.org/partpdf/232926

\section{Holding Institution}

MBLWHOI Library

\section{Sponsored by}

MBLWHOI Library

\section{Copyright \& Reuse}

Copyright Status: NOT_IN_COPYRIGHT

This document was created from content at the Biodiversity Heritage Library, the world's largest open access digital library for biodiversity literature and archives. Visit BHL at https://www.biodiversitylibrary.org. 